\title{
¿Qué tan bien representados están los mamíferos mexicanos en la red federal de áreas naturales protegidas del país?
}

\author{
How well represented are Mexican mammals in the natural protected area network?
}

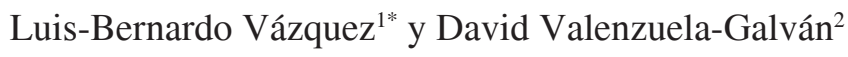 \\ ${ }^{1}$ El Colegio de la Frontera Sur, Departamento de Ecología y Sistemática Terrestres, Área Conservación de la Biodiversidad, Carretera Panamericana \\ y Periférico Sur $s / n, 29290$. San Cristóbal de Las Casas, Chiapas, México. \\ ${ }^{2}$ Departamento de Ecología y Conservación de los Recursos Naturales, Centro de Educación Ambiental e Investigación Sierra de Huautla (CEAMISH), \\ UAEM. Av. Universidad 1001, Col. Chamilpa, 62210. Cuernavaca, Morelos, México. \\ *Correspondencia: l.b.vazquez@gmail.com,lbvazquez@ecosur.mx
}

\begin{abstract}
Resumen. Se evaluaron la eficiencia y eficacia de la red de áreas naturales protegidas (ANPs) federales de México para representar la riqueza de especies de mamíferos terrestres. Para determinar el porcentaje de especies no protegidas, se sobrepuso una gradilla de celdas de $0.5^{\circ}$ x $0.5^{\circ}$ con datos de riqueza de especies en el mapa de las ANPs y se comparó con conjuntos equivalentes de áreas seleccionados al azar. Mediante optimización se estimó cuántas celdas adicionales complementarían a las ANPs y cuántas áreas bastarían para proteger todas las especies al menos una vez. Los resultados indican que cerca de la quinta parte del total de especies, un tercio de las endémicas y un tercio de las vulnerables, no están protegidas. En conjuntos de celdas elegidos al azar se representan más especies que en el total de celdas cubiertas por ANPs. En la península de Baja California, la vertiente del Pacífico, la sierra Madre Occidental y el sur del país (Oaxaca) se concentra el mayor número de especies no protegidas. Para complementar las celdas con ANPs se requieren 25 celdas adicionales y bastarían conjuntos óptimos de 38 celdas para representar todas las especies al menos una vez. Nuestros resultados pueden ayudar a mejorar la selección de nuevas ANPs para ganar en eficacia y eficiencia al representar a las especies de mamíferos aún no protegidas.
\end{abstract}

Palabras clave: conservación de mamíferos, México, representatividad, complementariedad.

\begin{abstract}
We evaluated the efficacy and efficiency of the federal network of natural protected areas (NPAs) to represent the species richness of terrestrial mammals. We overlapped a $0.5^{\circ} \times 0.5^{\circ}$ grid with data on species richness with the map of NPAs, to determine the proportion of unprotected species. We compared this with equivalent sets of cells, chosen randomly. In addition, through optimization we determined how many additional cells will complement the NPAs and how many cells will represent all species at least once. We found that nearly $1 / 5$ of the total species, $1 / 3$ of the endemics and $1 / 3$ of the threatened species are unprotected. The sets of cells randomly chosen represent more species than the totality of grid cells with NPAs studied. The Baja California Peninsula, Pacific Slope, the Sierra Madre Occidental, and the south of the country (Oaxaca) concentrate the highest number of unprotected species. We found that to complement the grid cells with NPAs 25 additional cells are needed and that a set of 38 cells chosen optimally will be enough to represent all species at least one time. Our results can help to improve the selection of new NPAs to gain in efficacy and efficiency in the representation of still unprotected species.
\end{abstract}

Key words: mammal conservation, Mexico, representativeness, gap analysis, complementarity.

\section{Introducción}

El crecimiento poblacional humano ha impulsado la conversión de grandes extensiones de hábitats naturales en áreas para usos urbanos y agropecuarios, entre otros (Chapin et al., 2000; Laurance et al., 2001). Los hábitats naturales han sido alterados y fragmentados prácticamente en todo

Recibido: 12 noviembre 2007; aceptado: 05 diciembre 2008 el mundo (WRI, 2000), teniendo como consecuencia la extinción regional y global de numerosas especies y un dramático decremento de las poblaciones locales (Hughes, et al., 1997; Brooks et al., 1999; Ceballos y Ehrlich, 2002; Gaston, et al., 2003).

Se ha reconocido que el establecimiento de áreas protegidas es una herramienta potencialmente efectiva en la reducción de los impactos de tales amenazas antropogénicas (Bruner et al., 2001), y es vital para la preservación de la biodiversidad en muchas de las estrategias de conservación 
a escala regional y global (Margules y Pressey, 2000; Rodrigues et al., 2004). Actualmente, hay en el mundo alrededor de 102000 áreas protegidas que cubren aproximadamente 18.9 millones de $\mathrm{km}^{2}$ (ca. el $12.7 \%$ de la superficie terrestre; Chape et al., 2003). La gran mayoría de estas reservas fueron designadas durante el siglo pasado y probablemente constituyen el cambio de uso del suelo más grande en la historia (Chape et al., 2005).

En México, el concepto de área natural protegida (ANP) se conoce desde la época precolombina (Ordoñez-Díaz y Flores-Villela, 1995; Gómez-Pompa y Kaus, 1999). Sin embargo, formalmente la primer área natural protegida fue creada hasta 1876(Vargas, 1984). Desde entonces a la fecha se han establecido un gran número de ANPs, aumentando de 23 en 1932 (que protegían el 1\% del territorio nacional) a 161 en 2007, que representan el $11.56 \%$ de la superficie del país, según datos de la Comisión Nacional de Áreas Naturales Protegidas (CONANP: http://www.conanp.gob. mx/anp/anp.php; consultada el 6 de noviembre de 2007).

Esta cifra, cercana al objetivo de conservación del $10 \%$ de territorio de cada país, acordado en la Convención sobre Diversidad Biológica (SCBD, 2004), podría sugerir que en México el nivel de protección de la biodiversidad es adecuado. Sin embargo, cuando se revisan algunas de las características de las áreas protegidas del país, se hacen evidentes algunos aspectos que apuntan a la necesidad de aumentar el número de reservas y la superficie que cubren en el país.

Por ejemplo, con una extensión del $40 \%$ dentro del sistema nacional de áreas protegidas, los hábitats áridos y semiáridos del país serían los mejor representados dentro del sistema. Sin embargo, esto está determinado en su mayor parte por el establecimiento de 3 grandes áreas naturales protegidas: Valle de Cirios y El Vizcaíno (en la península de Baja California) y El Pinacate-Gran Desierto de Altar (en Sonora), que en conjunto tienen una superficie total de 5729424 ha. Pese su gran extensión y relevancia, las zonas áridas de otras regiones, tales como Tamaulipas y Querétaro, están pobremente representadas en el conjunto de ANPs del país.

Otros ambientes, cómo la selva baja caducifolia, tienen una representación extremadamente pobre dentro del conjunto de ANPs del país y sólo en algunas reservas pequeñas y muy dispersas entre sí. Muchas de estas reservas albergan especies amenazadas y de distribución restringida, pero su tamaño y la proximidad a grandes centro urbanos dificultan la viabilidad y permanencia de estas especies a largo plazo (Vázquez y Gaston, 2006).

En general, la mayoría de las áreas protegidas en México son reservas ad hoc que no fueron originalmente establecidas con el objetivo de conservar la diversidad biológica (Ordoñez-Díaz y Flores-Villela, 1995; Simonian,
$1995)$ y por ello muchas no están localizadas en los mejores sitios para la protección de la diversidad biológica(e.g. sitios de mayor riqueza o mayor concentración de endemismos). Asimismo, la planeación y los fondos para su manejo eran limitados o simplemente no existían (Flores-Villela y Gerez, 1994). Como consecuencia, es predecible que una gran proporción de la biodiversidad del país permanezca sin protección alguna o esté pobremente representada dentro de la superficie actualmente protegida.

Uno de los primeros pasos para evaluar la eficacia de las ANPs en la protección de la biodiversidad es investigar el grado de representatividad de algunos grupos biológicos dentro del conjunto de ANPs del país (Rodrigues et al., 2004; Chape et al., 2005). Para México, algunos autores han hecho contribuciones importantes en esta dirección, reconociendo que el actual sistema de áreas protegidas es insuficiente para lograr un adecuado nivel de conservación de varios grupos biológicos presentes en el país (FloresVillela y Gerez, 1994, Ordoñez-Díaz y Flores-Villela, 1995; Cantú et al., 2004; Pérez-Arteaga, 2005; Ceballos, 2007). No obstante estos esfuerzos, el grado de representatividad de la biodiversidad dentro de las áreas protegidas es casi desconocido para la mayoría de los grupos taxonómicos presentes en el país (e.g., ¿cuántas especies del total del grupo de interés se protegen en alguna o algunas de las ANPs?).

Como una contribución a la solución de este problema, el objetivo del presente estudio fue evaluar la eficacia (e.g., ¿cuántas especies de mamíferos están o no representadas?) y eficiencia (e.g., ¿se representan de la mejor manera en términos de costos y resultados?) del conjunto de ANPs existente para proteger a los mamíferos Mexicanos. Para ello se determinó la proporción de especies no protegidas en estas áreas y esta representatividad se comparó con la alcanzada por un modelo nulo, así como con escenarios alternativos de conservación generados mediante análisis de complementariedad. Adicionalmente, se identificaron las zonas del país donde se concentran más especies no protegidas.

\section{Materiales y métodos}

Debido a que la información sobre la mastofauna presente en las ANPs es insuficiente, se usaron los datos generados previamente por Vázquez (2006) sobre el número e identidad de especies de mamíferos presentes en cada cuadro de una gradilla de 823 celdas de $0.5^{\circ} \mathrm{x}$ $0.5^{\circ}$, que cubre el país en su totalidad. Así, se siguieron como criterio taxonómico y punto de referencia de la distribución geográfica de cada especie, los mapas de distribución geográfica histórica de los mamíferos 
terrestres (excluyendo especies insulares e introducidas) presentes en el país, disponibles en el sitio de la Comisión Nacional para el Conocimiento y Uso de la Biodiversidad (CONABIO, http://www.conabio.gob.mx/informacion/ mamiferos/doctos/presentacion.html), a partir de los cuales Vázquez (2006) obtuvo el valor de riqueza de mamíferos para cada celda.

Esta gradilla fue sobrepuesta a los polígonos de las áreas naturales protegidas existentes en México tomados a partir de la versión 2005 de la base de datos mundial sobre áreas protegidas (WDPA por sus siglas en inglés; WDPA Consortium, 2005), que incluye las ANPs consideradas a esa fecha por la CONANP, y de esta manera se obtuvieron los datos de la riqueza de especies para cada celda que se sobrepusiera con las áreas protegidas, utilizando para ello herramientas de ArcView 3.2a (ESRI, 2000; para análisis similares véase Andelman y Willig, 2003; Rodrigues et al., 2003, 2004).

De las áreas protegidas consideradas dentro del listado utilizado en este trabajo sólo se tomaron en cuenta las áreas protegidas terrestres clasificadas en las categorías: Área de Protección de Flora y Fauna, Monumento Natural, Parque Nacional, Reserva de la Biosfera y otras categorías. En total, se tomaron en cuenta 63 ANPs con esas características, las cuales representan c. $61 \%$ de las ANPs terrestres reconocidas por la CONANP.

Los polígonos de estas ANPs se traslapan con un total de 163 celdas de medio grado, cubriendo entre 0.2 y $100 \%$ de cada celda (el traslape promedio de estas 163 celdas con las ANPs es de $30.56 \% \pm 33.48$ DE y el valor de la mediana es de 14\%).

Para evaluar la representatividad que los mamíferos terrestres tienen en las celdas protegidas, se evaluó cuántas especies se encuentran en estas celdas, suponiendo que el porcentaje de área cubierta por 1 o varias ANPs tuviera las mismas características que el resto de la celda en la cual está localizada (Cuadro 1).

Para propósitos de discusión del análisis sólo se consideraron aquellas celdas que tuvieran $\geq 12 \%$ de su superficie cubierta por alguna o algunas de las 63 ANPs antes señaladas. Consideramos este valor un tanto arbitrario, porque se notó que a partir de este nivel mínimo de protección el número de especies protegidas caía por debajo del $90 \%$ del total de especies (Cuadro 1). Por otra parte, entre la totalidad de las ANPs, la CONANP reconoce un subgrupo que denomina Sistema Nacional de Áreas Naturales Protegidas (SINAP; http://www.conanp. gob.mx/sinap/), en el que sólo se incluyen áreas que por su biodiversidad y características ecológicas son de especial relevancia para el país, que tienen personal e instalaciones, programas de manejo, designación de políticas de uso y financiamiento para su operación y que por ello tienen
Cuadro 1. Número de especies protegidas y el porcentaje que representan del total de especies consideradas (entre paréntesis), al considerar únicamente celdas con diferentes niveles de protección, desde 0.2 hasta $100 \%$, lo que paulatinamente reduce el número de celdas consideradas protegidas a celdas con un porcentaje progresivamente mayor de protección

\begin{tabular}{ccc}
\hline $\begin{array}{c}\text { Nivel de } \\
\text { protección (\%) }\end{array}$ & Número de celdas & $\begin{array}{c}\text { Especies } \\
\text { protegidas }(\%)\end{array}$ \\
\hline 100 & 22 & $186(43.9)$ \\
$>75$ & 28 & $257(60.7)$ \\
$>50$ & 40 & $312(73.7)$ \\
$>25$ & 61 & $317(74.9)$ \\
$>12$ & 92 & $370(88.2)$ \\
$>6$ & 127 & $389(91.9)$ \\
$>3$ & 147 & $391(92.4)$ \\
$>1$ & 162 & $395(93.6)$ \\
$>0.2$ & 163 & $395(93.6)$ \\
\hline
\end{tabular}

mayor probabilidad para cumplir con el objetivo de conservar su biodiversidad (CONANP, 2002). Por ello, además, para cada celda protegida, identificamos si su protección se debía a un ANP dentro del SINAP o no.

Con el fin de evitar confusiones sobre la influencia del área con otros efectos, omitimos de nuestros análisis celdas con $\geq 25 \%$ de superficie cubierta por mar o compartida con otro país. Así, nuestro análisis se restringió a un total de 423 especies de mamíferos terrestres presentes en México en un total de 705 celdas de $0.5^{\circ} \times 0.5^{\circ}$, de las que 93 se consideraron protegidas. Entre estas últimas, 70 tienen ANPs incluidas en el SINAP (en adelante celdas SINAP; Fig. 1).

Análisis de la eficacia y eficiencia de las celdas protegidas. Se evaluó la eficacia de las celdas consideradas como protegidas y de las celdas SINAP para conocer la representación de todas las especies de: (i) mamíferos terrestres considerados; (ii) endémicas, definidas como aquéllas cuya distribución estuviera restringida exclusivamente al territorio mexicano; (iii) las incluidas en la lista roja de la IUCN (//www.redlist.org [consultada en diciembre 2006]), y (iv) las incluidas en la lista mexicana de especies amenazadas (SEMARNAT, 2002).

Se calculó el número de veces que cada especie está representada en las celdas protegidas como una medida gruesa del número mínimo de poblaciones que se están protegiendo en las ANPs del país que se consideraron y se evaluó el porcentaje del total del área de distribución de cada especie en el país que representan esas celdas.

Para determinar las zonas del país con mayor concentración de especies no protegidas, se obtuvo la suma de estas especies por celda y se desplegó esta información en mapas. 


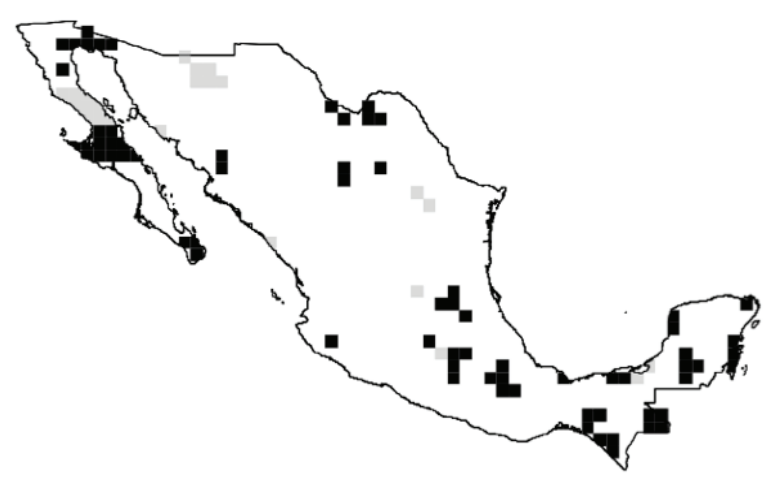

Figura 1. Distribución de las celdas protegidas en el país. En negro, las celdas SINAP; celdas grises + celdas negras representan las celdas protegidas.

La eficiencia de las celdas protegidas y de sólo las celdas SINAP, primero se determinó comparando qué tan bien representados están los mamíferos en estas celdas protegidas y en un modelo nulo equivalente a un número similar de celdas elegidas al azar (Rapoport et al., 1986; Burgess et al., 2002). Esto es, se evaluó si las reservas ya existentes (e.g. celdas protegidas o celdas SINAP), representan más especies, o no, que los conjuntos equivalentes de celdas elegidas al azar.

En este ejercicio se generaron mil conjuntos de celdas al azar, cada uno con el mismo número de celdas que el total de celdas protegidas o sólo celdas SINAP. Después se estimó el promedio de especies no representadas en estos modelos nulos y su intervalo de confianza al $95 \%$ y este dato fue comparado con el número de especies no representadas en las celdas protegidas o las celdas SINAP.

Después, se llevó a cabo un análisis de complementariedad con procedimientos de optimización (e.g. un proceso de selección de áreas que garantiza que cada nueva selección dé la mayor ganancia posible en términos de especies no representadas en las áreas ya seleccionadas; véase Underhill, 1994, Justus y Sarkar, 2002); por una parte, para determinar el conjunto mínimo de celdas que se requieren para complementar las ANPs y llegar a la meta más simple en términos de representatividad, que todos los mamíferos estén protegidos al menos una vez (e.g. en al menos una celda protegida) y, por otra parte, para determinar el conjunto mínimo de celdas (e.g. conjunto óptimo) que se requieren para lograr el mismo objetivo, sin considerar las ya protegidas. Los conjuntos óptimos de celdas complementarias se generaron con programación lineal en el programa C-PLEX 7.1 (ILOG, 2001).

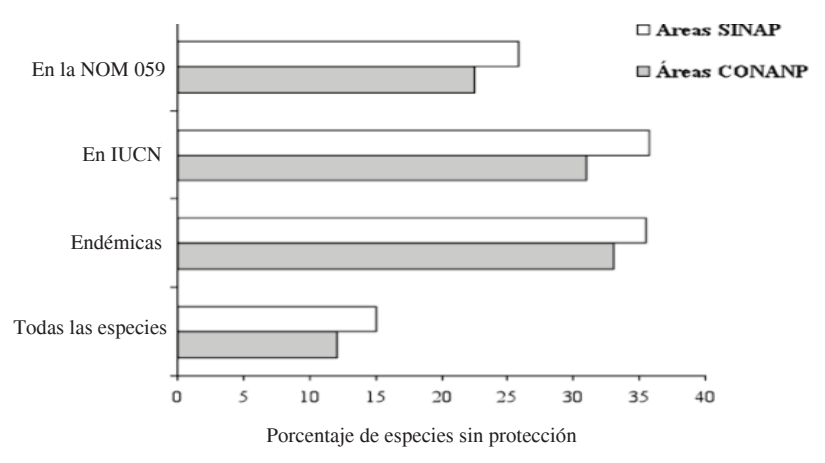

Figura 2. Porcentaje de mamíferos no representados en las celdas protegidas y sólo las celdas SINAP.

\section{Resultados}

La eficacia del sistema nacional de áreas protegidas. En las 93 celdas protegidas que se consideraron, se representa al menos una vez a 373 de las 423 especies de mamíferos terrestres presentes en el país (88.2\% del total) y si sólo se consideran las 70 celdas SINAP, el número de especies protegidas se reduce a 359 (84.9\% del total; Fig. 2). Las mayores omisiones de protección se localizaron principalmente en la península de Baja California, el occidente (Colima, Jalisco y Michoacán) y el sur del país (Oaxaca, Fig. 3a), y además en la región noreste del país entre Sonora y Chihuahua, al considerar sólo las celdas SINAP (Fig. 4a).

Al tomar en cuenta todas las celdas protegidas, se representa en promedio 20.3 veces (i.e., número de celdas protegidas; DE 18.7) y el $18.7 \%(\mathrm{DE} 14.5 \%$ ) del área de distribución total de cada una de las 373 especies sí protegidas en el país. Cuando sólo se tomaron en cuenta las celdas SINAP, las 359 especies representadas,están en un promedio de 16.6 celdas protegidas (DE 14.2), y esto equivale a que el $16.2 \%$ (DE $14.0 \%$ ) de la distribución geográfica total en el país de cada una de estas 359 especies se protege.

Al considerar las especies endémicas (121) se encontró que el 33 y $35.5 \%$ no están representadas en las celdas protegidas o sólo en las celdas SINAP, respectivamente (Fig. 2). Las regiones con mayor número de especies endémicas sin protección son la vertiente del Pacifico, destacando las costas de Jalisco, Colima y Michoacán, la parte central del país la península de Baja California y la sierra Madre Occidental (Figs. 3b y 4b).

En el caso de las especies mexicanas listadas en alguna de las categorías de amenaza de la IUCN, el $31 \%$ de las 42 especies no estuvieron representadas en ninguna celda protegida, cifra que aumenta al $35.8 \%$ al considerar sólo las 
a

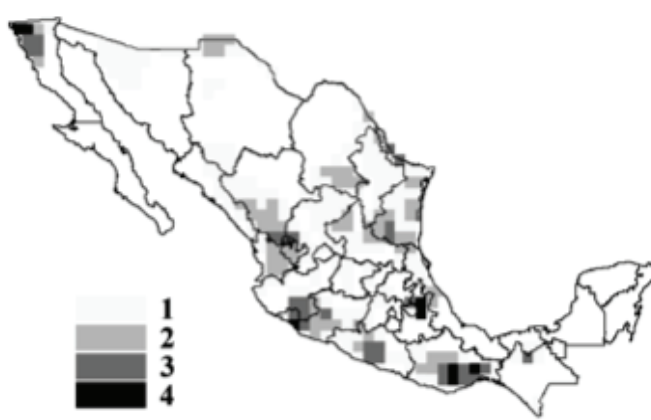

b

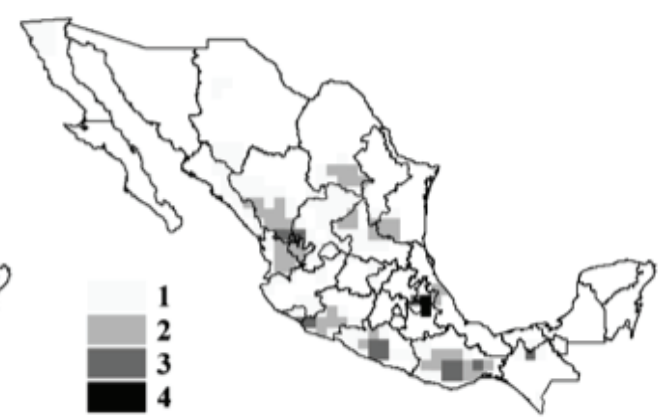

d

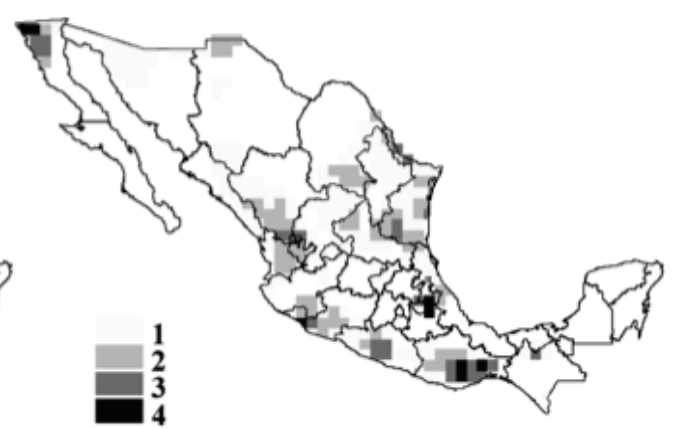

Figura 3. Densidad de especies de mamíferos terrestres de México no representadas en las celdas protegidas: a, todas las especies; b, endémicas; c, amenazadas (IUCN); d, en la NOM-059.

celdas SINAP (Fig. 2). Si tomamos en cuenta las especies registradas en la lista mexicana de especies amenazadas, 27 especies ( $22.5 \%$ de la lista total) no están protegidas y esto aumenta a 31 especies (25.8\% del total) considerando únicamente las celdas SINAP (Fig. 2).

En este caso, los mayores vacíos para los 2 grupos de especies amenazadas se registraron a lo largo de la vertiente del Pacífico, en el eje neovolcánico, sierra Madre del Sur, sierra Madre Occidental y el norte de la península de Baja California (Figs. 3c-d y 4c-d).

La eficiencia del sistema nacional de áreas protegidas. Cuando la representatividad de las especies de mamíferos en las celdas protegidas y celdas SINAP se comparó con los conjuntos de celdas equivalentes seleccionados al azar se encontró que en promedio estos conjuntos, representan mejor la riqueza de especies para todos los subconjuntos de mamíferos analizados que los 2 conjuntos de celdas evaluadas con áreas naturales protegidas (Cuadro 2).

Con el ejercicio de complementariedad, se encontró que se requieren conjuntos de 25 celdas adicionales a las protegidas para lograr la representación de todas las especies de mamíferos terrestres, al menos una vez. Así, en total se necesitarían 118 celdas de $0.5^{\circ} \times 0.5^{\circ}$, incluyendo las celdas protegidas, para lograr la representación de la totalidad de la mastofauna terrestre del país.

Teóricamente, se requerirían conjuntos de sólo 38 celdas (seleccionados de un total de 92 celdas) para representar a todos los mamíferos terrestres del país al menos una vez. Este número es considerablemente menor que las 93 celdas que se tomaron en cuenta en este trabajo como protegidas por alguna de las ANPs del país y que no representa, ni una sola vez, el $11.8 \%$ de los mamíferos terrestres. Este resultado también es más eficiente que la representatividad de especies alcanzada por los conjuntos de celdas elegidos al azar.

Además hay una baja coincidencia entre los escenarios de conservación analizados y los conjuntos óptimos obtenidos en el ejercicio de complementariedad. Por ejemplo, en el ejercicio para representar todas las especies una sola vez, únicamente 18 celdas (de un total de 92) en los conjuntos mínimos complementarios coinciden con celdas que contienen alguna área CONANP y sólo 17 celdas coinciden con celdas con áreas SINAP. 
a

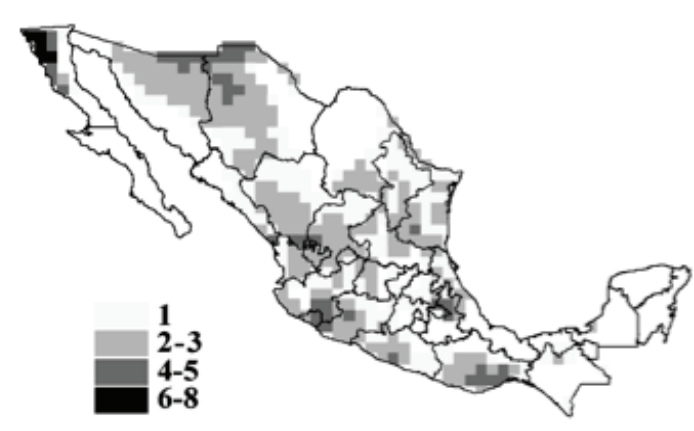

c

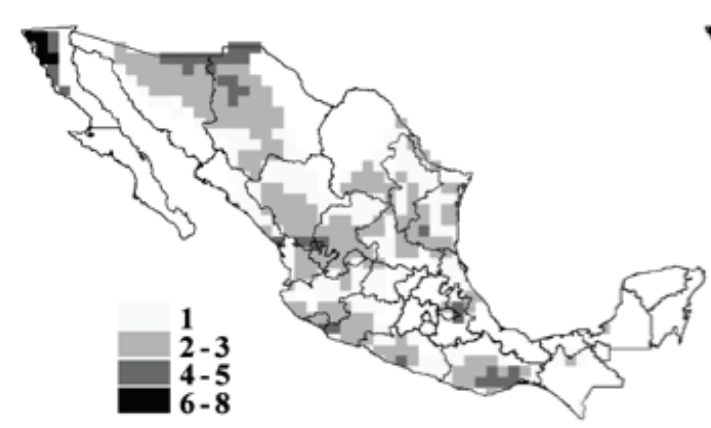

b

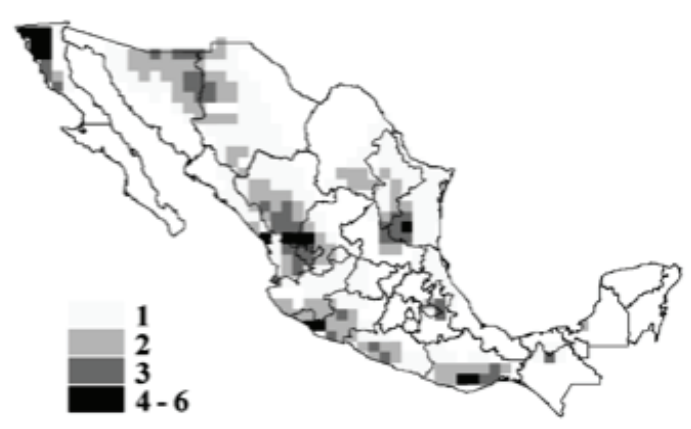

d

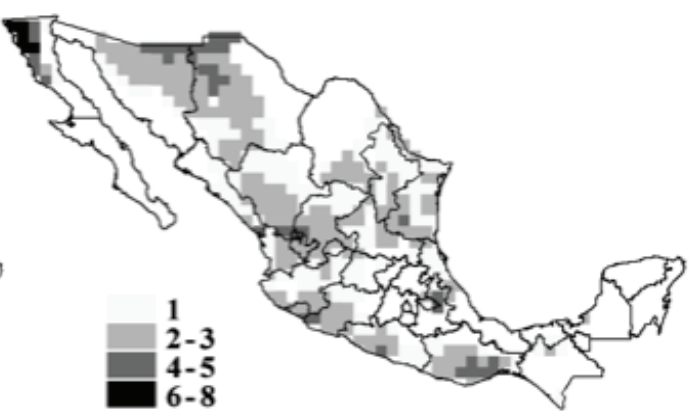

Figura 4. Densidad de especies de mamíferos terrestres de México no representadas en las celdas SINAP: a, todas las especies; b, endémicas; c, amenazadas (IUCN), y d, amenazadas (NOM-059).

\section{Discusión}

Los resultados presentados en este trabajo tienen importantes implicaciones para la planeación de estrategias de conservación en el país. Muestran que el actual sistema de áreas protegidas representa a más del $84 \%$ de la mastofauna, pero aún hay cerca de una quinta parte del total de especies no protegidas; entre las que se cuenta cerca de una tercera parte de las especies endémicas y las especies consideradas vulnerables por la IUCN presentes en el país y cerca de una cuarta parte de las especies consideradas vulnerables nacionalmente. Existe poca coincidencia entre las áreas protegidas y la presencia de un gran número de especies.

Esto es similar a los resultados registrados previamente en otros trabajos. Por ejemplo, Cantú et al. (2004) evaluaron la relevancia de las ANPs y de las zonas propuestas para conservación de México, para proteger su diversidad biológica y diferentes características geofísicas relevantes. Encontraron que las ANPs existentes son insuficientes para la protección de grupos importantes de especies y de algunas características, como los tipos de suelo o de vegetación; no obstante, esto puede mejorar notablemente si se establecen las ANPs que se plantean..

En otro trabajo, Ceballos (2007) evaluó la eficiencia para proteger la mastofauna de las 30 reservas más grandes de México, con inventarios completos, y encontró que un $82 \%$ de las especies de mamíferos están representadas al menos una vez en estas reservas y que se requerirían 25 celdas de $0.5^{\circ} \times 0.5^{\circ}$ para complementarlas y proteger a todos los mamíferos terrestres en al menos una reserva. En este sentido, nuestros resultados resaltan aún más que las ANPs existentes tienen una eficiencia relativamente baja, pues aún considerando un número sustancialmente mayor de ANPs que Ceballos (2007), la representatividad no aumenta mucho más ( $82 \%$ vs $84-88 \%$ en nuestro trabajo).

Por otra parte, nuestros resultados indican también que las especies que sí están representadas, lo están en un número razonable de áreas protegidas y se protege en promedio cerca de una quinta parte de su área de distribución en el país.

Pero es necesario considerar que se ha señalado que la mayoría de las ANPs en el país están situadas en regiones donde el promedio de la densidad poblacional humana 
Cuadro 2. Eficacia de las celdas protegidas (93) y las celdas SINAP (70) consideradas para representar a los mamíferos terrestres del país en comparación con la representatividad promedio en modelos nulos equivalentes. Se registran los valores del porcentaje del total de especies (423) y del total de endémicas (121), vulnerables según IUCN (42) y el gobierno mexicano (120), que sí están representadas en cada escenario; en el caso de los modelos nulos, se registra el porcentaje promedio de especies representadas al considerar sólo los conjuntos incluidos en los umbrales inferior y superior al $2.5 \%$

\begin{tabular}{lcccccccc}
\hline & $\begin{array}{c}\text { Celdas } \\
\text { protegidas }\end{array}$ & $\begin{array}{c}\text { Modelo } \\
\text { nulo }\end{array}$ & $\begin{array}{c}\text { Umbral } \\
\text { inferior }\end{array}$ & $\begin{array}{c}\text { Umbral } \\
\text { superior }\end{array}$ & $\begin{array}{c}\text { Celdas } \\
\text { SINAP }\end{array}$ & $\begin{array}{c}\text { Modelo } \\
\text { nulo }\end{array}$ & $\begin{array}{c}\text { Umbral } \\
\text { inferior }\end{array}$ & $\begin{array}{c}\text { Umbral } \\
\text { superior }\end{array}$ \\
\hline Total & 88.2 & 88.6 & 78.9 & 91.1 & 84.9 & 84.8 & 80.4 & 88.2 \\
Endémicas & 66.9 & 77.7 & 73.1 & 82.3 & 64.5 & 73.1 & 66.2 & 79.6 \\
Vulnerables & 69.1 & 73.7 & 67.7 & 80.1 & 64.2 & 69.8 & 43.5 & 78.2 \\
IUCN & 77.5 & 82.2 & 77.6 & 86.2 & 74.2 & 79.5 & 75.5 & 83.3 \\
$\begin{array}{l}\text { Vulnerables } \\
\text { lista mexicana }\end{array}$ & & & & & & & & \\
\hline
\end{tabular}

rebasa la media nacional (ca.50 personas $\mathrm{km}^{-2}$ ) y que el tamaño de las ANPs está negativamente correlacionada con la densidad poblacional humana (Vázquez y Gaston, 2006). Esto plantea la posibilidad de que varias de las ANPs existentes enfrenten cada vez más presión externa de las actividades humanas y por tanto puedan dejar de proteger adecuadamente algunas de las especies consideradas en este estudio.

Además, es importante tomar en cuenta el escenario más conservador resultante de considerar sólo a las ANPs incluidas en el SINAP, pues estas reservas son las que en la práctica podrían tener mayor probabilidad de ser eficientes a largo plazo. Con este trabajo se evidencia que la distribución de las ANPs incluidas en el SINAP, tiende a concentrarse en el sureste del país, lo que potencialmente tiene implicaciones importantes para la conservación de los mamíferos.

Por otra parte, los conjuntos equivalentes de áreas seleccionadas al azar representaron mejor el total de especies (i.e., fueron más eficientes) que las ANPs existentes, pero no que los conjuntos de celdas seleccionadas por complementariedad. Esto es similar a lo que se ha encontrado en otras partes del mundo (e.g. Burgess et al., 2005; De Klerk et al., 2004; Fjeldsa et al., 2004; Rebelo y Siegfried, 1992), lo que refleja el sesgo geográfico en la localización de muchas de las áreas protegidas, así como el que su distribución puede llegar a tener poca coincidencia con la distribución de la diversidad biológica.

Es importante señalar que estos resultados podrían diferir de manera importante con la realidad y con los de otros estudios por diferentes factores. Por una parte, los datos de distribución empleados son muy generales y tienden a sobrerrepresentar la distribución real de las especies que tienen patrones más complejos de distribución geográfica. Desde luego, todo proceso de complementariedad se ve afectado por la cantidad e identidad de especies a proteger, por lo que resultados como los aquí presentados se ven afectados por cambios en el criterio taxonómico o en la forma de estimar la distribución geográfica de cada especie.

En este sentido, el uso de datos de presencia, tanto de las especies como de las áreas protegidas dentro de una gradilla, como criterio para determinar si una especie está representada o no en una ANP, tiende a sobreestimar el número de especies que pueden considerarse protegidas. Por ello se trato de minimizar lo anterior, tomando como celdas protegidas sólo aquellas con $\geq 12 \%$ de su superficie cubierta por el polígono de alguna ANP, valor un tanto arbitrario, elegido porque a partir de este valor de protección el porcentaje del total de especies que se protegían caía por debajo del $90 \%$.

Además, este valor mínimo representa una superficie protegida superior a 30000 ha, lo que ya permite suponer la protección de poblaciones, incluso de especies con mayores necesidades de espacio, como es el caso de los grandes carnívoros cuya probabilidad de persistencia en una reserva se ve afectada de manera importante por el tamaño y forma de las ANPs (Woodroffe y Ginsberg, 1998).

Por otra parte, es necesario considerar que en algunas de las áreas elegidas en los conjuntos complementarios, la vegetación natural podría estar muy alterada o podrían tener una fuerte presión para el cambio de uso de suelo. Este es un aspecto que debería incorporarse con detalle al proceso de selección de áreas. De manera exploratoria se encontró que en las 92 celdas usadas para obtener los conjuntos complementarios de 38 celdas antes mencionados, el uso de suelo predominante va de vegetación natural no impactada a moderadamente impactada, y en promedio el $78.8 \%$, de cada una de estas celdas aún mantiene una buena cobertura forestal natural (rango de 20.6-100\%; Vázquez, 2006). 
Finalmente, el registrar una especie como presente o ausente dentro de las ANPs (e.g. celdas protegidas) no necesariamente implica que esté protegida adecuadamente; por ello, se calculó el número de poblaciones y porcentaje del área de distribución en el país que las ANPs podrían estar protegiendo, aunque esto requiere de estudios con más detalle.

Tomando en cuenta lo anterior, es probable que las regiones que concentran más especies no protegidas, detectadas en este estudio, puedan llegar a ser aún más evidentes y también que se destaquen otras zonas. Algunas de estas regiones, coinciden con zonas identificadas previamente como sitios prioritarios para la conservación (para varios grupos biológicos) por evaluaciones internacionales, entre las que sobresalen los hotspots (Mittermeier et al., 1999), áreas prístinas (Mittermeier et al., 2003) y centros de endemismo (Ramamoorthy et al., 1993; Myers et al., 2000). Otros estudios a escala nacional también han sugerido que los esfuerzos de conservación deberán concentrarse en estas regiones (Arita et al., 1997; Ceballos, 1999; Ceballos, et al., 1998; Ceballos, 2007, Cantú et al., 2004; Pérez-Arteaga, 2005; Peterson et al., 1993).

Desafortunadamente, es en varias de estas regiones donde la pérdida de hábitat y la deforestación comienzan a ser más severos y donde los efectos de la explotación son más grandes (Vázquez y Gaston, 2006). Un ejemplo claro es la selva baja caducifolia en las regiones occidente y suroeste del país. Este hábitat es rico en suelos para la agricultura (Brooks et al., 2004) y alberga un gran número de especies endémicas de mamíferos (Ceballos et al., 1998), así como de otros grupos (Gentry, 1995); hoy día, menos del $1 \%$ de su superficie original existe en el país y en su mayoría este tipo de hábitats cuenta con poca representación en los sistemas de áreas protegidas (Brooks et al., 2004).

Debido a que en general existe poca coincidencia entre los conjuntos complementarios y las celdas protegidas, es de destacar que la distribución geográfica de las ANPs del país no es la más adecuada para asegurar una eficaz protección para un importante número de especies de mamíferos presentes en México, por lo que es necesario buscar que la selección de nuevas ANPs mejore este problema, siguiendo procedimientos y objetivos claros que maximicen la representación de la biodiversidad dentro de las ANPs y que aseguren el buen uso de los escasos recursos disponibles para este tipo de actividades.

Desde luego los resultados aquí obtenidos pueden refinarse; entre los aspectos importantes que podrían incorporarse a la selección de celdas complementarias para la conservación de los mamíferos en el país estaría el buscar su conectividad, tomar en cuenta el costo de la tierra (e.g., costo potencial de una ANP en un sitio dado), la eficiencia de protección por unidad monetaria invertida, y qué tan factible es que en una celda seleccionada persista la vegetación natural sometida a transformación debido a la presión de actividades humanas o al cambio climático esperado (Balmford y Gaston, 1999; Briers, 2002; Gaston et al. 2002; Pyke et al. 2005; Fuller et al. 2006; Vázquez y Gaston, 2006; Escalante et al., 2007; Underwood et al. 2008).

Es necesario señalar que ciertas acciones de conservación deben realizarse en un plazo relativamente corto pues la eficiencia de los planes y propuestas de conservación decrece con el tiempo (Fuller et al., 2007).

Los resultados de este trabajo deben considerarse una guía indicativa de dónde podría ser más eficiente invertir en la creación de ANPs para conservar los mamíferos del país, más que una propuesta definitiva. Valorar el establecimiento de nuevas ANPs tomando en cuenta estos resultados implica un trabajo adicional y un análisis más detallado que el que aquí se ha empledo. Será muy recomendable definir un marco de inversión que permita incorporar datos socioeconómicos y políticos a una escala fina y llevar a cabo estrategias dirigidas a minimizar las amenazas para la conservación (Wilson et al. 2007). Consideramos que los resultados que se presentan y los procedimientos de selección empleados pueden ayudar a lograr este objetivo.

\section{Agradecimientos}

Agradecemos a M. de la Peña y X. López sus revisiones a diferentes versiones de este artículo. La mayor parte de su elaboración se dio durante una estancia de investigación de LBV en el CEAMISH-UAEM y una estancia sabática de DVZ en el Laboratorio de Ecología y Conservación de Fauna Silvestre del Instituto de Ecología, UNAM; por ello agradecemos a los doctores L. Durand y G. Ceballos, y al CEAMISH-UAEM por el apoyo brindado durante estas estancias.

\section{Literatura citada}

Andelman, S. J. y M. R Willig. 2003. Present patterns and future prospects for biodiversity in the Western Hemisphere. Ecology Letters 6:818-824.

Arita, H. T. y G. Ceballos. 1997. Los mamíferos de México, distribución y estado de conservación. Revista Mexicana de Mastozoología 2:33-71.

Balmford, A. y K. J. Gaston. 1999. Why biodiversity surveys are good value. Nature 398:204-205. 
Briers, R. 2002. Incorporating connectivity into reserve selection procedures. Biological Conservation 103:77-83.

Brooks, T. M., M. I. Bakarr, T. Boucher, G.A.B. Da Fonseca, C. Hilton-Taylor, J. M. Hoekstra, S. Olivieri, J. Parrish, R. L. Pressey, A. S. L. Rodrigues, W. Sechrest, W. A. Stattersfield, W. Strahm y S. N. Stuart. 2004. Coverage provided by the global protected-area system: Is it enough? Bioscience 54:1081-1091.

Bruner, A. G., R. E. Gullison, R. E. Rice y G. A. B. Fonseca. 2001. Effectiveness of parks protecting tropical biodiversity. Science 291:125-128.

Burgess, N., W. Kuper, J. Mutke, J. Brown, S. Westaway, S. Turpie, C. Meshack, J. Taplin, C. McClean y J. C. Lovett. 2005. Major gaps in the distribution of protected areas for threatened and narrow range Afrotropical plants. Biodiversity and Conservation 14:1877-1894.

Burgess, N. D., C. Rahbek, F.W. Larsen, P. Williams y A. Balmford. 2002. How much of the vertebrate diversity of sub-Saharan Africa is catered for by recent conservation proposals? Biological Conservation 107:327-339.

Cantú, C., R. G. Wright, J. M. Scout y E. Strand. 2004. Assessment of current and proposed nature reserves of Mexico based on their capacity to protected geophysical features and biodiversity. Biological Conservation 115:411-417.

Ceballos, G. 1999 Conservación de los mamíferos de México. Biodiversitas (Boletín de la Comisión Nacional para el Conocimiento y Uso de la Biodiversidad) 27:1-8.

Ceballos, G. 2007. Conservation priorities for mammals in megadiverse Mexico: the efficiency of reserve networks. Ecological Applications 17:569-578.

Ceballos, G., J. Arroyo-Cabrales y R. A. Medellín. 2002. The mammals of Mexico: composition, distribution, and conservation status. Occasional Papers of the Museum Texas Tech University218:1-28.

Ceballos, G. y P. R. Ehrlich. 2002. Mammal population losses and the extinction crisis. Science 296:904-907.

Ceballos, G., P. Rodríguez y R. A. Medellín. 1998. Assessing conservation priorities in megadiverse Mexico: mammalian diversity, endemicity, and endangerment. Ecological Application 8:8-17.

Chape, S., S. Blyth, L. Fish, P. Fox y M. Spalding. 2003. United Nations list of protected areas. UNEP-World Conservation Monitoring Centre/IUCN-The World Conservation Union, Cambridge/Gland.

Chape, S., J. Harrison, M. Spalding y I. Lysenko. 2005. Measuring the extent and effectiveness of protected areas as an indicator for meeting global biodiversity targets. Philosophical Transactions of the Royal Society of London B 260:443-455.

Chapin, S. F., E. S. Zavaleta, V. T. Eviner, R. L. Naylor, P. M. Vitousek, H. L. Reynolds, D. U. Hooper, S. Lavorel, O. E. Sala, S. E. Hobbie, M. C. Mack y S. Díaz. 2000. Consequences of changing biodiversity. Nature 405:234-242.

CONANP (Comisión Nacional de Áreas Naturales Protegidas). 2002. Perspectivas y logros. Informe técnico en el 11 aniversario. Secretaría del Medio Ambiente y Recursos Naturales, México, D. F.

De Klerk, H. M., J. Fjeldså, S. Blyth y N. D. Burgess, N. D.
2004. Gaps in the protected area network for threatened Afrotropical birds. Biological Conservation 117:529-537.

Escalante, T., V. Sánchez-Cordero, J. J. Morrone y M. Linaje. 2007. Deforestation affects biogeographical regionalization: a case study contrasting potential and extant distributions of Mexican terrestrial mammals. Journal of Natural History 41:965-984.

ESRI. 2000. ArcView 3.2 GIS. ESRI technology.

Fjeldså, J., N. Burgess, S. Blyth y H. M. Klerk. 2004. Where are the major gaps in the reserve network for Africa's mammals? Oryx 38:17-25.

Flores-Villela, O. y P. Gerez, P. 1994. Biodiversidad y conservación en México. Comisión para el Conocimiento y Uso de la Biodiversidad/Universidad Nacional Autónoma de México, México, D.F.

Fuller, T., M. Mayfield, M. Munguía, V. Sánchez-Cordero y S. Sarkar. 2006. Incorporating connectivity into conservation planning: a multi-criteria case study from Central Mexico. Biological Conservation 133:131-142.

Fuller, T. M., V. Sánchez-Cordero, P. Illoldi-Rangel, M. Linaje y S. Sarkar, 2007. The cost of postponing biodiversity conservation. Biological Conservation 134:593-600.

Gaston, K., R. L. Pressey y C. R. Margules. 2002. Persistence and vulnerability: retaining biodiversity in the landscape and in protected areas. Journal of Bioscience 27:361-384.

Gaston, K. J., T. M. Blackburn y K. Goldewijk. 2003. Habitat conversion and global avian biodiversity loss. Proceedings of the Royal. Society of London B 270:1293-1300.

Gentry, A.H. 1995. Diversity and composition of Neotropical dry forests. In Seasonally dry tropical forest, S. H. Bullock, H. A. Mooney y E. Medina (eds.). Cambridge University Press. p. 146-194.

Gómez-Pompa A. y R. Dirzo. 1995. Las reservas de la biosfera y otras áreas naturales protegidas de México. SEMARNAT/ CONABIO, México, D.F.

Gómez-Pompa, A. y A. Kaus. 1999. From pre-Hispanic to future conservation alternatives: lessons from Mexico. Proccedings of the National Academy of Sciences 96:5982-5986.

Hughes, J. B., Daily, G.C. y P. R. Erhlich. 1997. Population density: its extent and extinctions. Science 278:689-692.

ILOG. 2001. CPLEX 7.1. ILOG, Gentilly.

Justus, J. y S. Sarkar. 2002. The principle of complementarity in the design of reserve networks to conserve biodiversity: a preliminary history. Journal of Bioscience 27:421-435.

Laurance, W. F., M. A. Cochrane, S. Bergen, P. M. Fearnside, P. Delamônica, C. Barber, S. D’Angelo y T. Fernández. 2001. The future of the Brazilian Amazon. Science 291:438-439.

Margules, C. R. y R. L. Pressey. 2000. Systematic conservation planning. Nature 405:243-253.

Mittermeier R. A., N. Meyers, P. Robles Gil y C.G. Mittermeier. 1999. Hotspots - Earth's biologically richest and most endangered terrestrial ecoregions. CEMEX/Conservation International, University of Chicago Press, Illinois.

Mittermeier R. A., C. G. Mittermeier, T. M. Brooks, J. D. Pilgrim, W. R. Konstant, G. A. B. da Fonseca y C. Kormos. 2003. Wilderness and biodiversity conservation. Proccedings of the National Academy of Sciences 100:10309-10313.

Myers, N., R. A Mittermeier, C. G. Mittermeier, G. A. B. 
da Fonseca y J. Kent. 2000. Biodiversity hotspots for conservation priorities. Nature 403:853-858.

Ordoñez-Díaz, M. de J., y O. Flores-Villela. 1995. Áreas Naturales Protegidas. Serie Cuadernos de Conservación 4. PRONATURA, México, D.F.

Peterson, A. T., O. Flores-Villela, L.S. León-Paniagua, J. LlorenteBousquets, A. Luis-Martínez, A.G. Navarro-Sigüenza, M. G. Torres-Chávez y I. Vargas-Fernández. 1993. Conservation priorities in México: moving in the world. Biodiversity Letters 1:33-38.

Pérez-Arteaga, A. 2005. Conservation planning for waterbirds in Mexico. Ph.D. Thesis. University of Sheffield.

Pyke, C. R. S.J. Andelman y G. Midgley. 2005. Identifying priority areas for bioclimatic representation under climate change: a case study for Proteaceae in the Cape Floristic Region, South Africa. Biological Conservation 125:1-9.

Ramamoorthy, T. P., R. Bye, A. Lot y J. Fa. 1993. Biological diversity of Mexico: origins and distribution. Oxford University Press, New York.

Rapoport, E. H., G. Boriolo, J. E. Momjeau, J. E. Puntieri y R. D. Oviedo. 1986. The design of nature reserve: a simulation trial for assessing specific conservation value. Biological Conservation 37:269-290.

Rebelo, A. G., y W. R. Siegfried. 1992. Where should nature reserves be located in the Cape Floristic region, South Africa? Models for the spatial configuration of a reserve network aimed at maximizing the protection of floral diversity. Conservation Biology 6:243-252.

Rodrigues, A. S. L., S. J. Andelman, M. I. Bakarr, L. Boitani, T. M. Brooks, R. M. Cowling, L. D. C. Fishpool, G. A. B. Fonseca, K. J. Gaston, M. Hoffman, J. Long, P.A. Marquet, J. D. Pilgrim, R. L. Pressey, J. Schipper, W. Sechrest, S. N. Stuart, L. G. Underhill, R. W. Waller, M. E. J. Watts y X. Yan. 2003. Global Gap Analysis: towards a representative network of protected areas. Advances in Applied Biodiversity Science 5. Washington DC: Conservation International.

Rodrigues, A. S. L., S. J. Andelman, M. I. Bakarr, L. Boitani, T. M. Brooks., R. M. Cowling, L. D. C. Fishpool, G. A. B. Fonseca, K. J. Gaston, M. Hoffman, J. Long, P. A. Marquet, J. D. Pilgrim, R. L. Pressey, J. Schipper, W. Sechrest, S. N. Stuart, L. G. Underhill, R. W. Waller, M. E. J. Watts y X. Yan. 2004. Effectiveness of the global protected area network in representing species diversity. Nature 428:640-643.

SCBD. 2004 (Secretariat of the Convention on Biological
Diversity).Decisions adopted by the Conference of the Parties to the Convention on Biological Diversity at its Seventh Meeting, Montreal. UNEP/CBD/COP/7/21. SCBD SEMARNAT. 2002. Norma Oficial Mexicana PROY-NOM059-ECOL-2001, Protección ambiental - especies nativas de México de flora y fauna silvestres - categorías en riesgo y especificaciones para su inclusión, exclusión o cambio - lista de especies en riesgo. Diario Oficial de la Federación.06 marzo, 2002.

Simonian, L. 1995. Defending the land of the jaguar: a history of conservation in Mexico. The University of Texas Press, Austin.

Underhill, L. G. 1994. Optimal and suboptimal reserve selection algorithms. Biological Conservation 70:85-87.

Underwood, E. C., M. R. Shaw, K. A. Wilson, P. Kareiva, K. R. Klausmeyer, M. F. McBride, M. Bode, S. A. Morrison, J. M. Hoekstra y H. P. Possingham. 2008. Protecting biodiversity when money matters: maximizing return on investment. PLoS ONE 3: 1-7. e1515. doi:10.1371/journal. pone. 0001515 .

Vargas, F. 1984. Parques nacionales de México y reservas equivalentes. Instituto de Investigaciones Económicas Universidad Nacional Autónoma de México, México, D.F.

Vázquez, L. B. 2006. Distribution patterns and conservation priorities of mammals in Mexico. Tesis doctorado, University of Sheffield, UK. 162 p.

Vázquez, L. B. y K. J. Gaston. 2006. People and mammals in Mexico: conservation conflicts at a national scale. Biodiversity and Conservation 15:2397-2414.

WDPA Consortium. 2005. World database on protected areas 2005. PNUMA-WCMC.

Wilson, K. A., E. C. Underwood, S. A. Morrison, K. R. Klausmeyer, W. W. Murdoch, B. Reyers, G. WardellJohnson, P. A. Marquet, P. W. Rundel, M. F. McBride, R. L. Pressey, M.Bode, J. M. Hoekstra, S. Andelman, M. Looker, C. Rondinini, P. Kareiva, M. R. Shaw y H.P. Possingham. 2007. Conserving biodiversity efficiently: what to do, where, and when. PLoS Biol 5: 1850-1861. e223. doi:10.1371/ journal.pbio.0050223.

WRI (World Resources Institute). 2000. World Resources 20002001. World Resources Institute,Washington D. C.

Woodroffe, R y J. R. Ginsberg. 1998. Edge effects and the extinction of populations inside protected areas. Science 280:2126-2128. 